\title{
Probing IGM large-scale flows: warps in galaxies at shells of voids
}

\author{
M. López-Corredoira ${ }^{1}$, E. Florido ${ }^{2}$, J. Betancort-Rijo ${ }^{1,3}$, I. Trujillo ${ }^{1,4}$, C. Carretero ${ }^{1,5}$, A. Guijarro ${ }^{2,6}$, \\ E. Battaner ${ }^{2}$, and S. Patiri ${ }^{1,7}$ \\ 1 Instituto de Astrofísica de Canarias, C/.Vía Láctea, s/n, 38200 La Laguna (S/C de Tenerife), Spain \\ e-mail: martinlc@iac.es \\ 2 Departamento de Física Teórica y del Cosmos, Universidad de Granada, Spain \\ 3 Departamento de Astrofísica, Universidad de La Laguna, Tenerife, Spain \\ 4 School of Physics and Astronomy, University of Nottingham, University Park, Nottingham NG7 2RD, UK \\ 5 Estin \& Co Strategy Consulting, 43 Av. de Friedland, 75008 Paris, France \\ ${ }^{6}$ Centro Astronómico Hispano Alemán, Almería, Spain \\ 7 Case Western Reserve University, Cleveland (Ohio), USA \\ Received 11 June 2007 / Accepted 6 June 2008
}

\section{ABSTRACT}

\begin{abstract}
Context. Hydrodynamical cosmological simulations predict flows of the intergalactic medium along the radial vector of the voids, approximately in the direction of the infall of matter at the early stages of the galaxy formation.

Aims. These flows might be detected by analysing the dependence of the warp amplitude on the inclination of the galaxies at the shells of the voids with respect to the radial vector of the voids. This analysis will be the topic of this paper.

Methods. We develop a statistical method of analysing the correlation of the amplitude of the warp and the inclination of the galaxy at the void surface. This is applied to a sample of 97 edge-on galaxies from the Sloan Digital Sky Survey. Our results are compared with the theoretical expectations, which are also derived in this paper.

Results. Our results allow us to reject the null hypothesis (i.e., the non-correlation of the warp amplitude and the inclination of the galaxy with respect to the void surface) at $94.4 \%$ C. L., which is not conclusive. The absence of the radial flows cannot be excluded at present, although we can put a constraint on the maximum average density of baryonic matter of the radial flows of $\left\langle\rho_{\mathrm{b}}\right\rangle\left\langle\sim 4 \Omega_{\mathrm{b}} \rho_{\text {crit }}\right.$.
\end{abstract}

Key words. intergalactic medium - galaxies: statistics - galaxies: kinematic and dynamics - large-scale structure of Universe

\section{Introduction}

Warps seem to be an almost universal structural feature in spiral galaxies. Indeed, most of the spiral galaxies for which we have relevant information on their structure (because they are edge on and nearby) present warps in their stellar and gas distributions. Sánchez-Saavedra et al. (1990, 2003) and Reshetnikov \& Combes (1998) show that nearly half of the spiral galaxies of selected samples are warped, and many of the rest might also be warped since warps in galaxies with low inclination are difficult to detect. They are more clearly observed in the HI distribution (see e.g. van der Kruit 2007 and references therein). Warps are also detected at about $z=1$, even with a larger amplitude (Reshetnikov et al. 2002).

Despite the compelling observational evidence of warps in the spiral discs, there is not consensus on what could be the origin of this property of the galaxies. Nevertheless, it seems clear that warps should be produced by an interaction of the disc with an external element. In fact, Hunter \& Toomre (1969) showed that in an isolated galaxy (without a dark matter halo), an initial warp would soon disappear and leave as its only trace a thickening of the edge of the disc.

The number of ideas suggested to explain the origin of the warp in discs is vast. One explanation for the warps is gravitational tidal effects due to satellite galaxies. At least in the Milky Way galaxy, this explanation does not work with Magellanic Clouds as satellite (Hunter \& Toomre 1969), and it is controversial whether it works in combination with the amplification of the halo (as proposed by Weinberg 1998 and criticised by García-Ruiz et al. 2002). Also, the intergalactic magnetic field has been suggested as the cause of galactic warps (Battaner et al. 1990, 1991; Battaner \& Jiménez-Vicente 1998).

Following the evidence that galaxies seem to be embedded in a massive dark matter halo, the interaction between the halo and the disc was explored. Ideas like 'dynamical friction' between the disc and a spherical halo (Bertin \& Mark 1980; Nelson \& Tremaine 1995), a flattened halo misaligned with the disc (Toomre 1983; Dekel \& Shlosman 1983; Sparke \& Casertano 1988; Kuijken 1991), or resonant interactions with a triaxial halo (Binney 1981) were explored. All these ideas, however, were rejected when the dark matter halo was modelled correctly as a deformable mass of collisionless particles, rather than as a rigid body (Binney et al. 1998). Since a warp represents a misalignment of the disc's inner and outer angular momentum, Ostriker \& Binney (1989) and Jiang \& Binney (1999) proposed a model in which warps are generated through accretion of material into the halo with a misaligned spin that changes the major axis of the halo with respect to the disc and consequently produces a torque over the disc. There is a need for substantial accretion of low angular momentum material from the IGM into the galaxies (Fraternali et al. 2007), and the direction of the net angular-momentum vector of the material that is currently being accreted should be constantly changing (Quinn \& Binney 1992).

Also based on infalling of material, but with a much weaker dependence on halo properties, some works 
(Mayor \& Vigroux 1981; Revaz \& Pfenninger 2001; LópezCorredoira et al. 2002; Sánchez-Salcedo 2006) have proposed a mechanism for the formation of the warp in terms of the infall of a very low density intergalactic medium onto the disc without the dynamical intervention of an intermediate halo. Both $S$-type and $U$-type warps can be produced by this interaction (López-Corredoira et al. 2002; Saha \& Jog 2006). Even if there are other mechanisms able to produce warps, at least we know that the infall of material onto the disc will always produce warps.

If the infall of material is relevant to the formation of the warp of the disc, the orientation of the galaxies within the cosmological large-scale structure where they are embedded should have an effect on the formation of these features. There is growing evidence that disc galaxies are not oriented randomly, but their angular momentum primarily point parallel to the filaments (or sheets) where they are located. In the supergalactic plane, there is a hint of an excess of galaxies whose angular momentum lie in this plane (Kashikawa \& Okamura 1992; Navarro et al. 2004). Beyond the local universe, Trujillo et al. (2006) show at the $99.7 \%$ level that spiral galaxies located on the shells of the largest cosmic voids $\left(r>10 h^{-1} \mathrm{Mpc}\right)$ have rotation axes that lie primarily on the void surface. Paz et al. (2008) point out that the angular momentum of flattened spheroidals in SDSS galaxies tends to be perpendicular to the large-scale structure. These alignments are expected to be a consequence of the gain in angular momentum of the galaxies at the early stages of their formation, when both the baryonic component and the dark matter protohalo are suffering tidal torques from neighbouring fluctuations. Using N-body simulations, the alignments of the angular momentum of the haloes with the large-scale distribution have been also found (Porciani et al. 2002; Bailin \& Steinmetz 2005; Brunino et al. 2007; Aragón-Calvo et al. 2007; Hahn et al. 2007; Paz et al. 2008).

The aim of this paper is to check whether the orientation of the spiral galaxies in the void surfaces is related to the presence of a warp or not. In contrast to filaments (which are strongly affected by redshift-space distortion), large cosmological voids are a feature easy to characterise from the observational point of view. In addition, another important advantage of the void scheme is that (because of the radial growing of the voids) the vector joining the centre of the void with the galaxy position is a good approximation of the direction of the maximum compression of the large-scale structure at that point. Consequently, the radial vector of the void at the galaxy position approximately represents the direction of the infall of matter at the early stages of the galaxy formation. At later epochs, however, most of the accretion of material in the galaxy is expected to be through the filaments (i.e. parallel to the void surface). According to López-Corredoira et al. (2002), the infall of material should produce a correlation between the orientation of the galaxy and the amplitude and direction of the $S$-component, or the $U$-component or both of them. We want to check this hypothesis here. The aim of this paper is producing a method for analysing the relationship of the warp amplitude in galaxies with the inclination of the galaxy with respect to the line "centre of void"-galaxy (to check the early accretion of material). This method is then applied to the edge-on galaxies and void catalogue used by Trujillo et al. (2006) for available images from Sloan Digital Sky Survey (SDSS) survey. The work presented here is an attempt to observationally characterise the influence of the large-scale structure (and, consequently, the cosmic infall of material) on the formation of the warps. Some works have previously dealt with no random orientations of warps on large

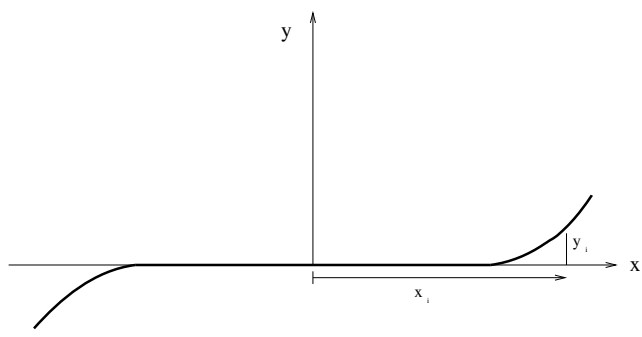

Fig. 1. Graphical representation of the warp measurement.

scales (Battaner et al. 1991) or in the Local Group (Zurita \& Battaner 1997), but not at the void shells.

\section{Definitions}

\subsection{Warp amplitude}

To define a warp amplitude, we first rotate the galaxy to have the mean plane of the galaxy coincident with the constant declination axis in the local plane of the sky (perpendicular to the line of sight). The position angle is calculated with an iterative method that fits the central part of galaxies (size of galaxy/2) to a straight line. This method uses the position angle from Trujillo et al. (2006) as starting point. The position angle was determined in this way to an accuracy of about 0.5 degrees. This error was adopted like that of the rms using the mean least square method in the rotation procedure. We then have a right and a left part of the galaxy, each having its own warp. The right part is the one with a lower right ascension. To quantitatively estimate the warp amplitude we define the warp parameter $W$, on the left(l) or right(r) side of the galaxy, as

$W_{(\mathrm{r} \text { or } 1)}=\frac{\int_{0}^{L_{(\mathrm{r} \text { or } \mathrm{l})}} x y \mathrm{~d} x}{\left(\int_{-L_{1}}^{L_{\mathrm{r}}} \mathrm{d} x\right)^{3}}$

where $L_{(\mathrm{r} \text { or } 1)}$ is the radius (left or right) of the disc within the limits in which the disc is visible $(\sigma \equiv$ signal/noise $>3$; $\sigma=\sqrt{\sigma_{\text {sys }}^{2}+\sigma_{\text {std }}^{2}}$, where $\sigma_{\text {sys }}$ is the systematic error and $\sigma_{\text {std }}$ the standard deviation) and $y$ is the height of the disc at position of pixel $x_{i}$, being $x \geq 0$ (Fig. 1). The y-values are obtained as the peaks of Gaussians fits in the light distribution perpendicular to the plane. As said, we only considered data with intensity greater than $3 \sigma$. An example of the result of our analysis is presented in Fig. 4, where the warp curve is drawn only for those values with an error bar less than $0.5^{\prime \prime}$. This estimated error bar can be computed by scaling the standard deviation ( $1 \sigma$ error) by the measured chi-squared value. Then, $W_{\mathrm{r}}$ will be positive for warp towards increasing declination, and vice versa for $W_{1}$. A large warp can reach values of $W=5 \times 10^{-3}$ and a barely perceptible warp $W=5 \times 10^{-4}$.

Expression (1) is adimensional, therefore the value of $W$ only depends on the shape of the edge-on galaxy but not on the intrinsic size or on the distance of a galaxy (neglecting the change of the factor $(1+z)^{4}$ (i.e. cosmological dimming) in the surface brightness of the galaxies throughout our sample since most of them are at a similar $z \sim 0.1$ ). For numerical purposes and working in pixels, we use the discrete expression

$W_{(\mathrm{r} \text { or } \mathrm{l})}=\frac{\sum_{0}^{N} x_{i} y_{i}}{\left(L_{\mathrm{l}}+L_{\mathrm{r}}\right)^{3}}$,

where $x_{i} \geq 0$ and $x_{N}=L_{(\mathrm{r} \text { or } 1)}$. The error in estimating $W$ is dominated by the imperfect rotation step of the galaxy when 

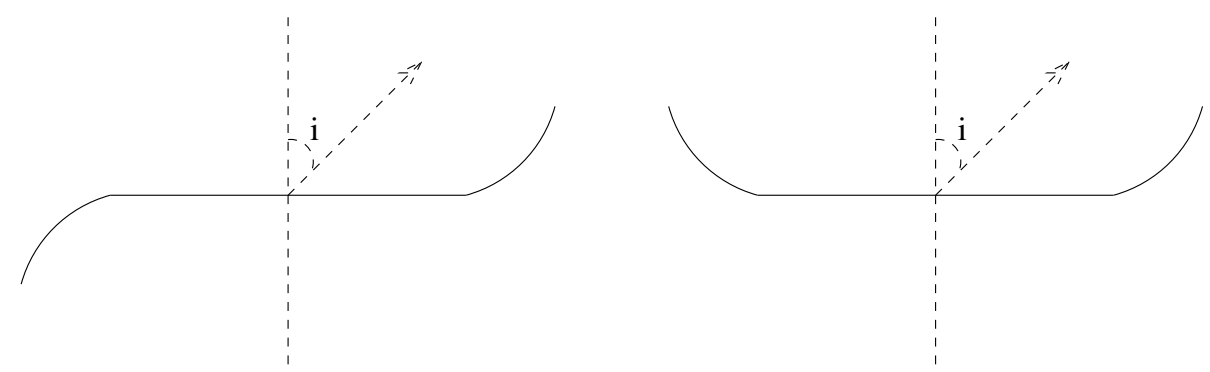

Fig. 2. Left: graphical representation of a perfect $S$-warp $(S \neq 0, U=0)$. Right: graphical representation of a perfect $U$-warp $(U \neq 0, S=0)$. North (higher declination) is up, south is down. "i" stands for the inclination between the line "centre of void"-galaxy and the rotation axis of the galaxy.

it is rotated to make the major axis coincident with the $\mathrm{x}$ axis. As mentioned before, this rotation is performed with an error of 0.5 degrees (i.e. about 0.008 radians). This introduces an error in $W$ given by

$\Delta W_{(\mathrm{r} \text { or } \mathrm{l})}=\frac{\int_{0}^{L_{\mathrm{(r} \text { or l })}} x^{2} \tan 0.008 \mathrm{~d} x}{\left(L_{\mathrm{r}}+L_{1}\right)^{3}} \sim 3.3 \times 10^{-4}$.

Further details of this method of warp measurement are given in Guijarro et al. (2008).

In a $S$-shape warped galaxy, $W_{\mathrm{r}}$ and $W_{1}$ have the same sign. In a $U$-shaped galaxy, $W_{\mathrm{r}}$ and $W_{1}$ have different signs. We define the variables $S$ and $U$ as

$S \equiv W_{\mathrm{r}}+W_{1}$,

$U \equiv W_{\mathrm{r}}-W_{1}$

If the warp is of the type with integral-sign [ $S$-warp, see Fig. 2 (left)], $S$ will be different from zero, positive or negative, and $U$ will be zero if it is perfectly symmetrical or has a low value if there is some asymmetry. Otherwise, if the warp is predominantly cup-shaped [ $U$-warp, see Fig. 2 (right)], $U$ will be different from zero and $S$ zero or very low, since it expresses the degree of asymmetry with respect to a perfect $U$-shape. An $L$-warp will have $|S| \approx|U|$. The combination of $S$-warps and $U$-warps explain the asymmetry of the warps (López-Corredoira et al. 2002; Saha \& Jog 2006) and the values of $S$ and $U$ give us the degree of each component to the total warp. We assign a value of $S$ and $U$ to all the galaxies in our sample and make statistics with these numbers, which quantify the $S$-component and the $U$-component.

A serious difficulty arising in any observational study of warps is that companions, spiral arms, and other effects may mimic warps. The errors introduced by a misidentification are difficult to evaluate. However, the images do not suggest that the warps are confused with spiral arms. On the other hand, the companions which are far away from the plane of the main galaxy are not confused with the warp, and if they were very close to the galactic outskirts, the galaxy would be removed from our list.

\subsection{Inclination of the galaxy with respect to the centre of the void}

For each galaxy, given its position angle and the position with respect to the centre of the void (see Trujillo et al. 2006 for details), we calculated the inclination of the rotation axis with respect to the line "centre of void"-galaxy. The sense of the rotation axis makes the "right" warp positive, that is, toward increasing declination. And the inclination $i$ is defined positive (between 0 and $\pi$ ) if the line "centre of void"-galaxy is to the right (decreasing position angle) of the rotation axis or negative (between 0 and $-\pi$ ) otherwise. Figure 2 illustrates this.

The error in this inclination stems from the error on the distance to the galaxies in Trujillo et al. (2006) sample. Due to the intrinsic motion of the galaxies away from the Hubble flow, this error is estimated to be around $4 h^{-1} \mathrm{Mpc}$, and the error in the distance to the centre of the void, around $2 h^{-1} \mathrm{Mpc}$. Taking into account that the average distance of the galaxies to the centre is $\approx 12 \mathrm{~h}^{-1} \mathrm{Mpc}$, this leads to an average error of $\approx 14^{\circ}$. Since these errors are statistical and not systematic, they will not affect the average signal that we find in the data, but will only decrease the signal-to-noise ratio.

\section{Theoretical predictions from a model of warp formation in terms of accretion of IGM onto the galactic disc}

\subsection{Warp dependence on inclination of the galaxy}

The predictions of the model with accretion of intergalactic medium (IGM) onto the disc for an average Milky Way-like galaxy is given in López-Corredoira et al. (2002, Fig. 11). A fit of the curves in that figure gives the theoretical values $S_{\mathrm{t}}$ and $U_{\mathrm{t}}$ :

$S$-component:

$S_{\mathrm{t}}(\theta[$ deg. $], v ; 0<\theta<90) \approx S_{0}(v)$

$\times \sin \left(4.99-1.26 \theta+0.164 \theta^{2}-0.00324 \theta^{3}+1.99 \times 10^{-5} \theta^{4}\right)$,

$S_{\mathrm{t}}\left(\theta[\right.$ deg.], $v ; 90<\theta<180)=-S_{\mathrm{t}}(180-\theta, v) ;$

$S_{\mathrm{t}}(\theta[$ deg. $], v ;-180<\theta<0)=S_{\mathrm{t}}(\theta+180, v)$

$U$-component:

$U_{\mathrm{t}}(\theta[$ deg. $], v ; 0<\theta<90) \approx U_{0}(v) \cos (1.16$

$\left.-0.295 \theta+0.0796 \theta^{2}-0.00173 \theta^{3}+1.09 \times 10^{-5} \theta^{4}\right)$,

$U_{\mathrm{t}}(\theta[$ deg. $], v ; 90<\theta<180)=-U_{\mathrm{t}}(180-\theta, v) ;$

$U_{\mathrm{t}}(\theta[$ deg. $], v ;-180<\theta<0)=U_{\mathrm{t}}(-\theta, v)$,

where $\theta$ in these expressions is the direction of the IGM wind with respect to the rotation axis of the galaxy, and $v$ the relative velocity of the wind. Together, $U_{0}$ and $S_{0}$ represent the maximum amplitude of the $S_{\mathrm{t}}$ and $U_{\mathrm{t}}$ that we calculate in the following sections. 

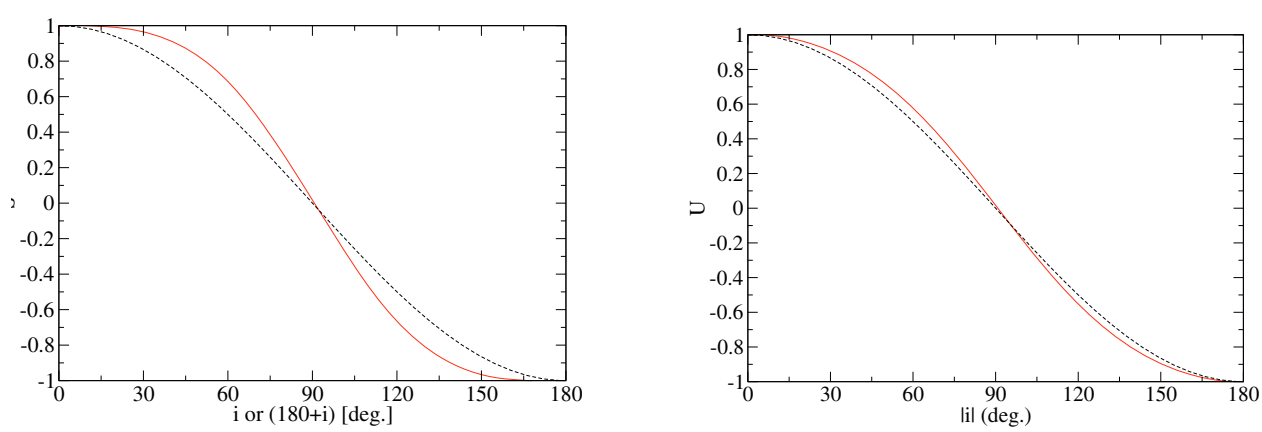

Fig. 3. Dependence of $\bar{S}$ and $\bar{U}$ on $i$ predicted by the theory. Solid line: expected trend if the warps were produced by intergalactic winds flowing radially outwards in the void, according to expressions (8) and (9), normalized to a maximum height of one. Dashed line: cos $i$.

Assuming there is a wind flowing radially outwards in the void with velocity $\overline{v_{1}}=200 \mathrm{~km} \mathrm{~s}^{-1}$ (details will be given in Betancort-Rijo \& Trujillo 2008), we must add the dispersion of velocities of the galaxies: $\sigma_{1}=215 \mathrm{~km} \mathrm{~s}^{-1}, \sigma_{2}=209 \mathrm{~km} \mathrm{~s}^{-1}$ (Betancort-Rijo \& Trujillo 2008) in the radial velocity $v_{1}$ (the projection of the velocity into the radial direction of the void) and the perpendicular component $v_{2}$ with respect to the radial direction of the void with angular azimuth $\phi$. To obtain these numbers, Betancort-Rijo \& Trujillo used the linear theory of growing fluctuations in the large-scale structure. They computed the r.m.s. of the corresponding components of the velocity of mass particles on the surface of a void of $10 h^{-1} \mathrm{Mpc}$ with respect to its centre of mass. These numbers agree within a few per cent with the numbers found in numerical simulations. Hence, the average warps are given by

$$
\begin{aligned}
& \overline{S_{t}}(i)=\frac{1}{\pi} \int_{0}^{\pi} \mathrm{d} \phi \int_{0}^{\infty} \mathrm{d} v_{2} \int_{-\infty}^{\infty} \mathrm{d} v_{1} P\left(v_{1}, v_{2}\right) S_{\mathrm{t}}(\theta, v), \\
& \overline{U_{t}}(i)=\frac{1}{\pi} \int_{0}^{\pi} \mathrm{d} \phi \int_{0}^{\infty} \mathrm{d} v_{2} \int_{-\infty}^{\infty} \mathrm{d} v_{1} P\left(v_{1}, v_{2}\right) U_{\mathrm{t}}(\theta, v), \\
& \theta=\cos ^{-1}\left(\frac{v_{1} \cos i+v_{2} \sin i \cos \phi}{v}\right) \\
& P\left(v_{1}, v_{2}\right)=\frac{1}{\sqrt{2 \pi} \sigma_{1}} \frac{v_{2}}{\sigma_{2}^{2}} \exp \left(-\frac{\left(v_{1}-\overline{v_{1}}\right)^{2}}{2 \sigma_{1}^{2}}-\frac{v_{2}^{2}}{2 \sigma_{2}^{2}}\right) \\
& v=\sqrt{v_{1}^{2}+v_{2}^{2}}
\end{aligned}
$$

where $S_{\mathrm{t}}(\theta, v) \propto v^{2}, U_{\mathrm{t}}(\theta, v) \propto v^{2}$ because the warp amplitude is proportional, both in the $S$-shape and $U$-shape, to $v^{2}$ (López-Corredoira et al. 2002; Eqs. (39), (45)). When we make all these calculations, we get a dependence of $S_{\mathrm{t}}(i)$ and $U_{\mathrm{t}}(i)$ on $i$ which is close to cos $i$ (see Fig. 3), although closer in the case of $U_{\mathrm{t}}(i)$ than in the case of $S_{\mathrm{t}}(i)$. Therefore, from now onwards, we will consider as a first-order approximation [here we include the amplitude resulting from the calculation with expressions (8), (9) approximately]:

$\overline{S_{\mathrm{t}}}(i) \approx 0.98 S_{0} \cos i$,

$\overline{U_{\mathrm{t}}}(i) \approx 1.18 U_{0} \cos i$.

To facilitate the comparison of our theory with the data, we estimate the correlations of $S_{\mathrm{t}}$ and $U_{\mathrm{t}}$ [full expressions (8), (9)] with the function $\cos i$.

$$
\begin{aligned}
& \left\langle\overline{S_{\mathrm{t}}} \cos i\right\rangle-\left\langle\overline{S_{\mathrm{t}}}\right\rangle\langle\cos i\rangle=0.53 S_{0}, \\
& \left\langle\overline{U_{\mathrm{t}}} \cos i\right\rangle-\left\langle\overline{U_{\mathrm{t}}}\right\rangle\langle\cos i\rangle=0.60 U_{0} .
\end{aligned}
$$

\subsection{Amplitude of the $S$-component: $S_{0}$}

From López-Corredoira et al. (2002, Figs. 10, 11, Eq. (39)), we can derive roughly that the maximum height $y$ of the $m=1 \mathrm{com}-$ ponent of warp of a Milky Way-like galaxy and baryonic mean density of the intergalactic medium $\rho_{\mathrm{b}}$ (roughly the average density of the IGM flows radially ejected from the void to produce the observed effect):

$$
y=2.8 \times 10^{18}{\overline{v_{1}}}^{2}\left(\mathrm{~km} \mathrm{~s}^{-1}\right) \rho_{\mathrm{b}}\left(\mathrm{kg} / \mathrm{m}^{3}\right) \exp [0.43 x(\mathrm{kpc})] \mathrm{kpc} .
$$

With the definition given in Eq. (1), $\overline{v_{1}}=200 \mathrm{~km} \mathrm{~s}^{-1}$, multiplying by a factor $2 / \pi$ for averaging the integration of the line of nodes over all the angles, the maximum amplitude is

$$
\begin{aligned}
\left|W_{(\mathrm{r} \text { or } 1)}\right|(\theta=0)= & \frac{2.1 \times 10^{22} \rho_{\mathrm{b}}\left(\mathrm{kg} / \mathrm{m}^{3}\right)}{L(\mathrm{kpc})^{3}} \\
& \times[(\exp [0.43 L(\mathrm{kpc})][L(\mathrm{kpc})-2.33])+2.33]
\end{aligned}
$$

The size (semiaxis length) of a Milky Way-like galaxy is approximately $L=15 \mathrm{kpc}$. With this number,

$\left|W_{(\mathrm{r} \text { or } \mathrm{l})}\right|(\theta=0) \sim 5 \times 10^{22} \rho_{\mathrm{b}}\left(\mathrm{kg} / \mathrm{m}^{3}\right)$.

That is, due to $S=2 W$,

$S_{0} \sim 10^{23} \rho_{\mathrm{b}}\left(\mathrm{kg} / \mathrm{m}^{3}\right)$

We must bear in mind that this is only an estimation of the order of magnitude, because not all the galaxies are like the Milky Way (although this is a reasonably good approximation for the galaxies in our sample). To give an example, with an average intergalactic medium density given by $\left\langle\rho_{\mathrm{b}}\right\rangle=\Omega_{\mathrm{b}} \rho_{\text {crit }} \sim$ $8 \times 10^{-28} \mathrm{~kg} / \mathrm{m}^{3}$ (taking $\Omega_{\mathrm{b}}=0.042$; Spergel et al. 2007), we get a $S$-component with $S_{0} \sim 8 \times 10^{-5}$.

\subsection{Amplitude of the U-component: $U_{0}$}

Similarly, from López-Corredoira et al. (2002, Figs. 10, 11, Eq. (45)), we derive roughly that the maximum height $y$ of the $m=0$ component of the warp of a Milky Way-like galaxy with IGM baryonic mean density $\rho_{\mathrm{b}}$ is

$$
y=8.2 \times 10^{17}{\overline{v_{1}}}^{2}\left(\mathrm{~km} \mathrm{~s}^{-1}\right) \rho_{\mathrm{b}}\left(\mathrm{kg} / \mathrm{m}^{3}\right) \exp [0.38 x(\mathrm{kpc})] \mathrm{kpc} .
$$

With the definition given in (1), $\overline{v_{1}}=200 \mathrm{~km} \mathrm{~s}^{-1}$, multiplying by a factor $2 / \pi$ for averaging the integration of the line of nodes over 

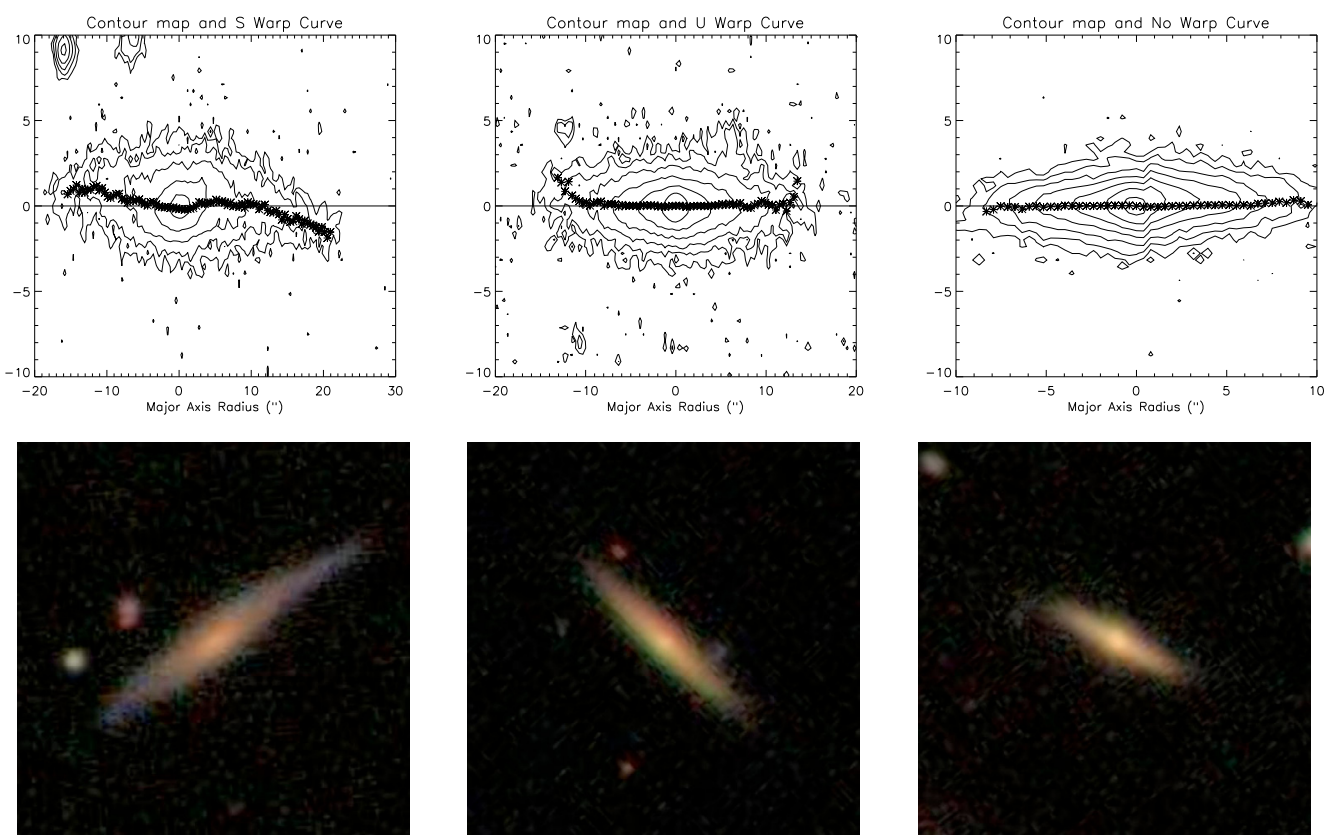

Fig. 4. Warp curves and contour maps of three selected galaxies $(u p)$; and 5 -filters combined SDSS images of them $\left(\right.$ down), $50^{\prime \prime} \times 50^{\prime \prime}$. The isophotes are equidistant (in units of $\mathrm{n}^{*} 3 \sigma$ equiv. to a step of $+0.75 \mathrm{mag} / \mathrm{arcsec}^{2}$ ) starting at a level of about $3 \sigma$ above the sky background. Left: $R A=234.8898^{\circ}, \delta=+3.2352^{\circ}(\mathrm{J} 2000), S$-warped. Centre: $R A=131.4264^{\circ}, \delta=+51.2122^{\circ}(\mathrm{J} 2000), U$-warped downwards. Right: $R A=226.3795^{\circ}, \delta=+57.4142^{\circ}(\mathrm{J} 2000)$, negligible warp.

the all angles, the maximum amplitude is

$\left|W_{(\mathrm{r} \text { or l })}\right|(\theta=0)=\frac{7.0 \times 10^{21} \rho_{\mathrm{b}}\left(\mathrm{kg} / \mathrm{m}^{3}\right)}{L(\mathrm{kpc})^{3}}$

$$
\times[(\exp [0.38 L(\mathrm{kpc})][L(\mathrm{kpc})-2.63])+2.63]
$$

With $L=15 \mathrm{kpc}$,

$\left|W_{(\mathrm{r} \text { or l })}\right|(\theta=0) \sim 0.8 \times 10^{22} \rho_{\mathrm{b}}\left(\mathrm{kg} / \mathrm{m}^{3}\right) ;$

that is, due to $U=2|W|$,

$U_{0} \sim 1.6 \times 10^{22} \rho_{\mathrm{b}}\left(\mathrm{kg} / \mathrm{m}^{3}\right)$.

Again, we call that this is a rough estimation with Milky Way-like galaxies. With an average intergalactic medium density given by $\left\langle\rho_{\mathrm{b}}\right\rangle=\Omega_{\mathrm{b}} \rho_{\text {crit }} \sim 8 \times 10^{-28} \mathrm{~kg} / \mathrm{m}^{3}$ (taking $\Omega_{\mathrm{b}}=0.042$; Spergel et al. 2007), we get a $U$-component with $U_{0}=1.3 \times 10^{-5}$.

\section{Data and analysis}

We used the data of the SDSS-DR3 (3rd. data release) that have already been used in Trujillo et al. (2006). These data are their edge-on (inclination larger than $78^{\circ}$ ) galaxies, which are within the shells $r_{\text {void }}<r<r_{\text {void }}+4 h^{-1} \mathrm{Mpc}$ surrounding the largest voids, where $r_{\text {void }}>10 h^{-1} \mathrm{Mpc}$ is its radius. The lower the inclination of the galaxy, the greater the thickness of the projected disc and, consequently, the greater the error in the determination of the centroid of $y(x)$. In the worst case $\left(78^{\circ}\right)$, the thickness of the projected disc is comparable to its intrinsic thickness (Dalcanton \& Bernstein 2002), so the error in the warp amplitude is not significantly increased with respect to a $90^{\circ}$ inclination galaxy.

The voids were located using maximal spheres empty of galaxies with magnitude over $-19.31-5 \log h\left(H_{0}=\right.$ $100 h \mathrm{~km} \mathrm{~s}^{-1} \mathrm{Mpc}$ ), and they were found by means of the HB void finder (Patiri et al. 2006). From the SDSS available public data, we used the filter " $r$ " images. In total we have 114 galaxies.

For seventeen galaxies there were difficulties measuring the warp amplitude (for instance, due to the proximity of a star in the field or interaction with other galaxies), so there remain $N=97$ galaxies with which we carried out the statistics (Table 1). In Fig. 4, we show three examples.

Some images of warped galaxies could be the subject of alternative interpretations. For instance, considering the isophote maps, warp curve, and image in the central panel of Fig. 4, a feature is found at $x=-12, y=5$, either a companion galaxy or an inteloper, which could produce/modify the warp curve. However, we find that the warp at this galactocentric radius is real, as directly deduced from a detailed study of the isophote maps.

If we plot their values of $S$ and $U$ vs. $i$, we get the results of Fig. 5. There are slight trends in $S(i)\left[\left\langle S i\left(^{\circ}\right)\right\rangle-\langle S\rangle\left\langle i\left(^{\circ}\right)\right\rangle=\right.$ $\left.\left(0.3^{\circ} \pm 6.1^{\circ}\right) \times 10^{-3}\right]$ and in $U(i)\left[\left\langle U i\left(^{\circ}\right)\right\rangle-\langle U\rangle\left\langle i\left(^{\circ}\right)\right\rangle=\left(-8.6^{\circ} \pm\right.\right.$ $\left.\left.5.6^{\circ}\right) \times 10^{-3}\right]$. The errors in the correlations are calculated as $\sigma_{S} \sigma_{i} / \sqrt{N}$ and $\sigma_{U} \sigma_{i} / \sqrt{N}$; where $\sigma_{S}, \sigma_{U}$ and $\sigma_{i}$ are the rms of the values of $S, U$, and $i$.

The scattering of Fig. 5 may be for several reasons. For example,

1. The measurement of the warp amplitude has errors.

2. Several mechanisms produce warps. The accretion produces signal and noise, while the other mechanisms only produce noise.

3. Different masses of the galaxies not taken into account in our model, as said in Sects. 3.2 and 3.3.

4. The wind's other components apart from the radial one introduce scattering.

5. The error of the inclination with respect to the void also introduces scattering.

All these sources of contamination introduce an increase in the scattering but not a systematic error. We can consider the error 

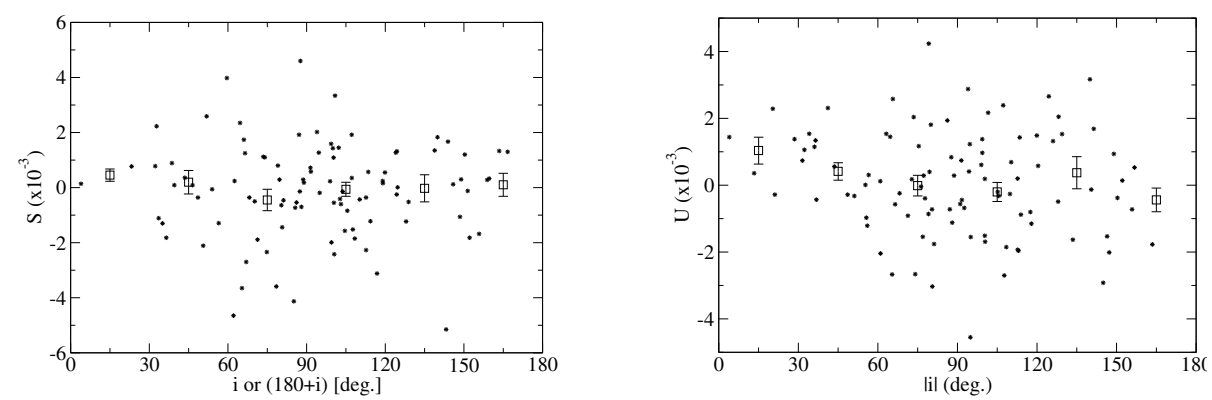

Fig. 5. Dependence of $S$ and $U$ on the inclination $i$ in the observational data (stars). The squares with error bars represent the average in bins of $i$ of 30 degrees.

of each individual point in Fig. 5 as $\sigma_{S}=1.7 \times 10^{-3}$ for the $S$-component and $\sigma_{U}=1.6 \times 10^{-3}$ for the $U$-component $(\mathrm{rms}$ in Fig. 5). Our mission is to extract the statistical information hidden behind these clouds of points.

\subsection{Checking the null-hypothesis}

To check whether the null hypothesis is compatible with the observed $U$-components distribution, we computed the probability, $P\left(i_{*}\right)$, based on the binomial distribution of finding no more than $n_{0}^{-}$galaxies with $U<0$ and $|i| \leq i_{*}$, and no more than $n_{1}^{+}$galaxies with $U>0$ and $|i| \geq 180-i_{*}$, assuming that there is no correlation between $i$ and $U$ (i.e., the null-hypothesis). With this assumption, the probability that $U>0$ is $1 / 2$ for any value of $i$, and using the binomial distribution for $n_{1}^{+}, n_{0}^{-}$, we find

$P\left(i_{*}\right)=\left(\sum_{i=0}^{n_{0}}\left(\begin{array}{l}m_{0} \\ i\end{array}\right)\right)\left(\sum_{i=0}^{n_{1}^{+}}\left(\begin{array}{l}m_{1} \\ i\end{array}\right)\right) 2^{-\left(m_{0}+m_{1}\right)}$,

where $m_{0}$ is the number of galaxies with $|i| \leq i_{*} ; m_{1}$ is the number of galaxies with $|i| \geq\left(180-i_{*}\right)$. To determine $i_{*}$, we assume that the signal is proportional to $\cos i$, as determined previously in our model [see Eq. (14)]. We assume that the galaxies are uniformly distributed in $i$ (the small alignment reported by Trujillo et al. 2006 is not very relevant to this purpose), then

$$
\langle\bar{U}\rangle\left(i_{*}\right)=\frac{1.18 U_{0} \int_{0}^{i_{*}} \sin i \cos i \mathrm{~d} i}{\int_{0}^{i_{*}} \sin i \mathrm{~d} i} \propto\left(1+\cos i_{*}\right) .
$$

The rms is proportional to the number of galaxies within $i<i_{*}$, which for a given sample and again assuming isotropy, has the proportionality

$\sigma_{\bar{U}}\left(i_{*}\right) \propto\left(1-\cos i_{*}\right)^{-1 / 2}$.

Thus, according to our model, the value of $i_{*}$ that maximizes the signal-to - noise ratio, $\frac{\langle\bar{U}\rangle}{\sigma_{\bar{U}}}$, is given for $i_{*}=60^{\circ}$. If instead of the approximate cosine dependence of Eq. (14), we took the exact calculation of Eq. (9), the value that maximizes the signal to noise ratio would be $i_{*}=73^{\circ}$ for $U$-component; for $S$-component, it would be $i_{*}=75^{\circ}$. We assume as a good approximation the cosine dependence, so we use $i_{*}=60^{\circ}$.

With this value of $i_{*}=60^{\circ}$, the probability that our data are compatible with the null hypothesis is $P=0.056$; that is, the null hypothesis is excluded within $94.4 \%$ C.L. If we took $i_{*}=45^{\circ}$, we would get $P=0.0043$ (rejection of non-correlation within $99.57 \%$ C.L.), but this value of $i_{*}$ is not justified, a priori; therefore, the statistical significance must be less than this. For a higher value of $i_{*}$, we also get rejection of the null hypothesis. For $i_{*}=75^{\circ}$ we get rejection within $95.7 \%$ C.L.
If we do the same calculation for the $S$ vs. $i$ data, we find that the probability of null hypothesis cannot be rejected $\left(P\left(i_{*}=60^{\circ}\right)=0.13\right)$. We also checked the null hypothesis with the Spearman rank correlation coefficient. This test gives higher probabilities of a null correlation: $P=0.154$ for $U(i)$ and $P=0.739$ for $S(i)$.

\subsection{Most likely values of the signal}

If we assume that Eq. (14) with some positive $U_{0}$ applies, the mean value of $U$ for $i \leq i_{*}, \bar{U}$, is given by Eq. (26):

$\bar{U}=0.88 U_{0}$

The most likely value of $\bar{U}$ can be estimated from the data as follows.

We assume that the distribution of the probabilities of a value of $U, P(U)$, is Gaussian, centred at $\bar{U}$ with r.m.s. $\sigma_{U}$. For galaxies with $i \leq i_{*}$, the probability that $U<0$ is

$\int_{-\infty}^{0} P(U) \mathrm{d} U \equiv \frac{1}{2}-\omega$

i.e.,

$\omega(U)=\frac{1}{2}\left(1-\operatorname{erfc}\left(\frac{U}{\sqrt{2} \sigma_{U}}\right)\right)$,

and the probability that $U>0$ is

$\int_{0}^{\infty} P(U) \mathrm{d} U=\frac{1}{2}+\omega$.

Identically, for $i \geq 180-i_{*}$ the probability that $U<0$ is $\frac{1}{2}+\omega$, and the probability that $U>0$ is $\frac{1}{2}-\omega$. The probability, $F(\omega)$, that the number of galaxies with $i \leq i_{*}$ and $U<0, k$, is smaller than or equal to the observed value $n_{0}^{-}$(from a total of $m_{0}$ galaxies with $i \leq i_{*}$ ) and that, for $i \geq 180-i_{*}$ the number of galaxies with $U>0, j$, is lower than or equal to the observed value, $n_{1}^{+}$ (from a total of $m_{1}$ galaxies with $i \geq 180-i_{*}$ ), is given by the multiplication of the probabilities of both events:

$$
\begin{aligned}
F(\omega) & =\left(\sum_{k=0}^{n_{0}}\left(\begin{array}{l}
m_{0} \\
k
\end{array}\right)(1 / 2+\omega)^{m_{0}-k}(1 / 2-\omega)^{k}\right) \\
& \times\left(\sum_{j=0}^{n_{1}^{+}}\left(\begin{array}{l}
m_{1} \\
j
\end{array}\right)(1 / 2+\omega)^{m_{1}-j}(1 / 2-\omega)^{j}\right) .
\end{aligned}
$$

Thus, the mean value $\bar{U}$ is obtained from $F[\omega(\bar{U})]=0.5$, and the maximum and minimum values within $95 \%$ C.L. would 
Table 1. Amplitude of the warp (with the corresponding error), in units of $10^{-3}$, and inclination with respect to the radial direction of the void of the used SDSS galaxies in this paper.

\begin{tabular}{|c|c|c|c|c|c|}
\hline$\overline{\mathrm{RA}\left({ }^{\circ}\right)}$ & $\overline{\text { Dec. }\left({ }^{\circ}\right)}$ & $\overline{W_{\mathrm{r}}}$ & $W_{1}$ & $\operatorname{Err}\left(W_{\mathrm{r} / 1}\right)$ & $\overline{i\left(\left(^{\circ}\right)\right.}$ \\
\hline 7.2201 & -10.1462 & -0.8 & -2.3 & 1.4 & -63.1 \\
\hline 8.1775 & -10.7132 & 0.1 & 0.5 & 1.7 & 91.6 \\
\hline 12.7354 & -10.3630 & -0.6 & 0.4 & 1.5 & -55.6 \\
\hline 16.2514 & -10.6816 & -0.4 & -0.1 & 2.5 & -51.1 \\
\hline 37.5906 & -1.1753 & 0.0 & 0.1 & 0.9 & -140.5 \\
\hline 44.4492 & -7.7039 & -0.8 & 0.9 & 1.5 & -133.5 \\
\hline 52.2561 & -0.2598 & 1.3 & -0.1 & 3.2 & -113.5 \\
\hline 116.3311 & 33.9423 & 0.0 & 0.8 & 0.7 & 79.1 \\
\hline 117.2566 & 40.5368 & -2.1 & 0.6 & 1.6 & 107.6 \\
\hline 118.8881 & 43.4200 & 2.3 & 1.7 & 2.3 & -120.4 \\
\hline 119.9286 & 44.3550 & 0.1 & -0.6 & 1.8 & 110.3 \\
\hline 120.5069 & 26.0021 & 0.5 & 0.9 & 2.4 & -77.7 \\
\hline 123.8789 & 41.7845 & 2.5 & -0.4 & 2.1 & 94.0 \\
\hline 126.8650 & 34.0610 & -1.0 & -1.4 & 2.4 & -79.5 \\
\hline 127.0763 & 30.4663 & 2.7 & 1.9 & 1.5 & 87.6 \\
\hline 127.3464 & 32.3032 & -0.1 & -0.3 & 0.5 & 112.7 \\
\hline 127.3562 & 34.7077 & 1.0 & 0.9 & 1.7 & -72.8 \\
\hline 128.1052 & 43.3659 & -2.3 & -0.4 & 1.4 & -113.0 \\
\hline 129.5621 & 46.6003 & 0.4 & -0.2 & 1.1 & 99.0 \\
\hline 129.9943 & 53.1409 & -0.9 & 0.7 & 1.8 & 95.0 \\
\hline 130.3608 & 43.7136 & -2.1 & 0.8 & 2.2 & -144.9 \\
\hline 131.4264 & 51.2122 & 0.7 & -1.9 & 0.7 & -65.6 \\
\hline 131.9592 & 46.4286 & 0.7 & 0.0 & 1.4 & 91.5 \\
\hline 133.3355 & 37.7273 & -2.5 & 0.5 & 3.9 & -80.5 \\
\hline 139.2459 & 3.2982 & -0.1 & -0.3 & 1.1 & -77.2 \\
\hline 139.4740 & 57.2313 & -0.2 & -1.6 & 1.5 & 36.5 \\
\hline 140.1681 & 46.3610 & -0.3 & -1.8 & 3.0 & -129.4 \\
\hline 140.9270 & 56.9905 & 2.0 & -0.7 & 0.8 & 124.4 \\
\hline 141.6051 & 46.8787 & 0.6 & 0.1 & 3.8 & -156.8 \\
\hline 143.5772 & 48.0993 & 0.8 & -0.6 & 1.1 & 4.0 \\
\hline 145.8396 & 1.7637 & 1.9 & 0.4 & 2.0 & 64.6 \\
\hline 148.3098 & 8.9179 & 0.5 & -0.1 & 1.1 & 43.6 \\
\hline 151.3779 & 5.6446 & 0.1 & 2.1 & 1.6 & -147.2 \\
\hline 151.6732 & 5.4257 & -0.2 & 1.3 & 1.9 & 100.3 \\
\hline 161.8397 & 65.1576 & -1.1 & 0.6 & 1.5 & 100.4 \\
\hline 162.2562 & 59.4725 & -0.7 & -2.9 & 0.9 & -101.6 \\
\hline 163.8244 & 48.8133 & 2.3 & 0.3 & 1.0 & -128.1 \\
\hline 164.7096 & 0.7530 & 1.4 & -1.0 & 2.8 & 107.2 \\
\hline 165.8762 & 50.9933 & 1.2 & 0.0 & 3.0 & 94.7 \\
\hline 167.3524 & 65.7939 & 0.9 & -0.1 & 0.9 & 32.3 \\
\hline 168.3881 & 58.0182 & 1.6 & -0.5 & 2.4 & 73.5 \\
\hline 172.3922 & 57.3641 & -2.8 & -2.4 & 1.8 & -36.8 \\
\hline 175.2166 & -2.8630 & -0.7 & 0.0 & 2.5 & 80.4 \\
\hline 176.9355 & 62.8054 & -0.8 & -1.0 & 0.7 & 152.2 \\
\hline 178.7274 & 1.3727 & -0.2 & -1.4 & 0.9 & -75.4 \\
\hline 180.3058 & 56.1816 & -0.5 & -0.8 & 0.8 & 56.5 \\
\hline 181.1288 & 54.6058 & -0.2 & 0.4 & 1.4 & -91.0 \\
\hline 181.5367 & 4.5987 & 0.4 & 1.3 & 1.2 & -113.9 \\
\hline 181.8765 & -2.0978 & -0.4 & -0.1 & 2.4 & -109.8 \\
\hline 185.1264 & -1.4101 & -0.3 & 0.5 & 2.1 & -117.5 \\
\hline 187.4005 & -3.7120 & 0.8 & -0.7 & 2.3 & -34.1 \\
\hline 187.4013 & -1.0802 & 0.4 & 0.8 & 1.3 & 150.3 \\
\hline 189.8933 & 5.0846 & -3.2 & -0.5 & 1.0 & 65.4 \\
\hline 191.5689 & 62.2786 & -0.9 & 0.2 & 1.7 & 88.0 \\
\hline 193.1906 & 62.6491 & -1.1 & 0.6 & 1.5 & 81.2 \\
\hline
\end{tabular}

be respectively $\bar{U}_{+}, \bar{U}_{-}$derived from $F\left[\omega\left(\bar{U}_{+}\right)\right]=0.95$, $F\left[\omega\left(\bar{U}_{-}\right)\right]=0.05$ respectively. Note that $\omega(U)$ has been defined so that it must be positive if our model applies. A negative value of $\omega(U)$ should be interpreted as evidence against it.

With our data and $i_{*}=60^{\circ}$, the values are: $\bar{U}=6.7 \times 10^{-4}$, $\bar{U}_{+}=14.5 \times 10^{-4}, \bar{U}_{-}=-0.3 \times 10^{-4}$. Using our model, we can use these numbers to put a constraint on the IGM density. From
Table 1. continued.

\begin{tabular}{cccccc}
\hline \hline RA & Dec. & $W_{\mathrm{r}}$ & $W_{\mathrm{l}}$ & $\operatorname{Err}\left(W_{\mathrm{r} / 1}\right)$ & $i\left(^{\circ}\right)$ \\
\hline 195.1273 & 0.4801 & -0.4 & 0.3 & 0.5 & -92.6 \\
195.7355 & 3.7370 & -1.3 & 0.2 & 1.4 & -146.4 \\
195.7591 & -2.4564 & -0.6 & -0.3 & 1.1 & 105.5 \\
197.1058 & 61.4598 & 0.6 & -0.7 & 0.9 & -126.0 \\
197.8032 & 49.4153 & 0.0 & -1.4 & 0.9 & -99.3 \\
198.0996 & -2.8867 & 1.6 & -0.2 & 1.4 & -80.0 \\
198.5669 & -2.0749 & 0.1 & 0.0 & 1.7 & -60.9 \\
198.9388 & 59.8330 & 0.8 & 0.5 & 1.1 & -13.3 \\
199.0415 & 65.9117 & -4.3 & 0.2 & 2.3 & -94.9 \\
204.4937 & 5.7839 & 0.6 & 1.3 & 2.3 & 87.1 \\
207.0692 & 46.7701 & -1.2 & -0.5 & 1.1 & 155.8 \\
207.7140 & 5.2116 & -0.9 & -0.4 & 1.3 & 128.0 \\
212.5481 & 58.7175 & 1.8 & -0.5 & 1.9 & -41.1 \\
214.8475 & 2.8468 & 2.5 & -0.7 & 1.5 & 139.9 \\
219.3559 & 0.4227 & 0.2 & 0.1 & 0.5 & -100.3 \\
220.0357 & 3.0825 & -0.3 & -0.1 & 0.3 & 68.2 \\
220.3983 & 62.7731 & 3.8 & -0.4 & 2.8 & -79.1 \\
220.7501 & 61.6237 & 1.0 & -0.5 & 1.6 & 119.9 \\
223.3199 & 49.1950 & -0.2 & 1.5 & 1.5 & 163.5 \\
225.0443 & 43.1852 & 0.6 & -0.3 & 1.6 & 148.9 \\
226.3795 & 57.4142 & 1.3 & 0.3 & 1.2 & 99.4 \\
226.8317 & 0.5954 & -1.3 & -1.1 & 1.1 & -105.2 \\
227.2538 & 41.3454 & -1.4 & -0.5 & 1.6 & 71.3 \\
230.8046 & 39.8048 & 0.7 & -1.2 & 1.5 & 86.2 \\
233.1236 & 50.0678 & -0.8 & 1.9 & 1.7 & 74.1 \\
233.7380 & 58.4997 & -0.3 & 0.0 & 0.8 & 48.6 \\
234.8898 & 3.2352 & -2.9 & -1.8 & 2.4 & -117.9 \\
236.9465 & 55.5457 & 0.6 & -0.8 & 1.5 & -28.5 \\
238.5349 & 45.0029 & 0.0 & 0.3 & 1.3 & -21.1 \\
239.2935 & 45.2622 & 0.3 & 0.0 & 0.7 & 88.7 \\
241.3029 & 42.8759 & -0.9 & 1.1 & 1.5 & -61.0 \\
242.0378 & 50.1382 & -0.1 & -0.1 & 1.2 & -76.3 \\
243.6710 & 38.0918 & -0.2 & -0.9 & 1.6 & -31.5 \\
244.8777 & 42.4485 & 1.3 & -1.0 & 1.7 & -20.4 \\
249.2328 & 44.0929 & 1.4 & 0.3 & 2.1 & -36.1 \\
251.6845 & 42.7894 & 0.0 & 1.2 & 1.9 & -56.0 \\
254.6449 & 32.6595 & 0.0 & 0.6 & 0.7 & -66.5 \\
257.1461 & 59.3407 & -0.2 & -0.6 & 0.7 & -94.4 \\
258.1745 & 30.4143 & -2.1 & -0.2 & 3.2 & 112.7 \\
324.0977 & -6.4763 & -1.9 & 0.0 & 0.8 & 108.4 \\
341.0004 & -0.9310 & 1.3 & -0.4 & 3.0 & -141.4 \\
354.9044 & 14.5762 & -1.1 & 0.5 & 1.3 & -76.9 \\
\hline & & & & &
\end{tabular}

Eqs. (28) and (24), we find that $\left\langle\rho_{\mathrm{b}}\right\rangle=4.8_{-4.9}^{+5.5} \times 10^{-26} \mathrm{~kg} / \mathrm{m}^{3}$ (95\% C.L.). This allows us to put an upper limit on the IGM density but not a minimum. The same calculation with $S$-component gives a tighter constrain: $\bar{S}=5.1 \times 10^{-4}, \bar{S}_{+}=13.6 \times 10^{-4}$, $\bar{S}_{-}=-2.3 \times 10^{-4} ;\left\langle\rho_{\mathrm{b}}\right\rangle=6.7_{-9.8}^{+11.4} \times 10^{-27} \mathrm{~kg} / \mathrm{m}^{3}$ (95\% C.L.).

The correlation of $S$ with a cosine function (approximately the expected shape theoretically) is $\langle S \cos i\rangle-\langle S\rangle\langle\cos i\rangle=$ $(-0.004 \pm 0.091) \times 10^{-3}$. The correlation of $U$ with $U_{\mathrm{t}}$ (also approximated to be a cosine function) is $\langle U \cos i\rangle-\langle U\rangle\langle\cos i\rangle=$ $(0.116 \pm 0.083) \times 10^{-3}$. We can get a better constraint for the maximum density from these correlations: with the $S$ component measurement and the expressions (15) and (20), we find that $\left\langle\rho_{\mathrm{b}}\right\rangle<\sim 3 \times 10^{-27} \mathrm{~kg} / \mathrm{m}^{3} \approx 4 \Omega_{\mathrm{b}} \rho_{\text {crit }}(95 \%$ C.L. $\equiv 2 \sigma)$; with the $U$-component measurement and the expressions (16) and (24), we find that $\left\langle\rho_{\mathrm{b}}\right\rangle<3 \sim 10^{-26} \mathrm{~kg} / \mathrm{m}^{3} \approx 37 \Omega_{\mathrm{b}} \rho_{\text {crit }}(95 \%$ C.L.).

Therefore, summarising the contents of this section, we reject the null hypothesis (i.e., the inclination of galaxies and the amplitude of the warp are not related to each other) at $94.4 \%$ 
C.L. Using our model, we can estimate the average density of the radial flow from the void to be $0-4\left(\frac{\overline{v_{1}}}{200 \mathrm{~km} \mathrm{~s}^{-1}}\right)^{-2} \Omega_{\mathrm{b}} \rho_{\text {crit }}$.

\section{Conclusions}

Cosmological hydrodynamical simulations predict flows of IGM along the radial vector of the void. This radial direction is approximately the same as the infall of matter in the early stages of the galaxy formation at the shells of the void. One way to search for the effect of this IGM flow in these shells is to measure the dependence of the warp amplitude on their galaxies as a function of their inclination with respect to the radial vector of the void. In this paper, we have developed a method to measure that effect, and we made a first attempt to find this effect. The signal found in the $U$-component of the warp (the null hypothesis is rejected at $94.4 \%$ C.L.) gives some hint that such an effect might exist. This result is not conclusive $(5.6 \%$ is not a very negligible probability) and the absence of the radial flows cannot be excluded at present. If the IGM radial flows in the radial direction of the voids exist, their baryonic matter density should be $\left\langle\rho_{\mathrm{b}}\right\rangle<\sim 3 \times 10^{-27} \mathrm{~kg} / \mathrm{m}^{3}=4 \Omega_{\mathrm{b}} \rho_{\text {crit }}$. This density would increase inversely proportional to the square of the mean flow velocity if its value differs from $200 \mathrm{~km} / \mathrm{s}$. There is also the possibility that the accretion of material have different initial velocities than the radial direction of the void.

There may be other mechanisms of warp formation different to the accretion onto the disc, but they would produce noise in the correlation if they have nothing to do with the IGM accretion. If the correlation of $S$-component amplitude and inclination were observed, although it would be an argument in favour of López-Corredoira et al. (2002) theory, it would not be totally conclusive because there might be alternative explanations for the correlation. The mechanism of accretion into the halo (Ostriker \& Binney 1989; Jiang \& Binney 1999) rather than onto the disc might possibly explain the correlation. There might be a relationship between warps and filaments associated to the void produced by primordial magnetic fields, or the frozen magnetic fields were aligned with the filaments (Florido \& Battaner 1997), if the magnetic fields are also responsible for the warp formation (Battaner et al. 1990, 1991; Battaner \& Jiménez-Vicente 1998). However, these theories do not explain the $U$-component (the asymmetry of the $S$-warps), which are clearly observed in many galaxies (e.g., Reshetnikov \& Combes 1998; Sánchez-Saavedra et al. 2003). The trend in the correlation of the $U$-component with the inclination of the galaxy obtained in this paper, if confirmed with higher statistical significance, could be taken as confirmation that the mechanism of IGM accretion onto the disc produces warps. The application of the method presented in this paper to galaxy samples with more objects and/or better measurements of the warp amplitude is expected to give more accurate results.

Acknowledgements. Thanks are given to the anonymous referee for helpful comments, and to Joly Adams (language editor of A\&A) for proof-reading this paper. Funding for the creation and distribution of the SDSS Archive has been provided by the Alfred P. Sloan Foundation, the Participating Institutions, the National Aeronautics and Space Administration, the National Science Foundation, the U.S. Department of Energy, the Japanese Monbukagakusho, and the Max Planck Society. The SDSS Web site is http://www.sdss.org/. The SDSS is managed by the Astrophysical Research Consortium (ARC) for the Participating Institutions. The Participating Institutions are The University of Chicago, Fermilab, the Institute for Advanced Study, the Japan Participation Group, The Johns Hopkins University, the Korean Scientist Group, Los Alamos National Laboratory, the Max-Planck-Institute for Astronomy (MPIA), the Max-Planck-Institute for Astrophysics (MPA), New Mexico State University, University of Pittsburgh, University of Portsmouth, Princeton University, the United States Naval Observatory, and the University of Washington. MLC was supported by the Ramón y Cajal Programme of the Spanish Science Ministery. We thank the Spanish Science Ministery for support under grant AYA200767625-CO2-01.

\section{References}

Aragón-Calvo, M. A., van de Weygaert, R., Jones, B. J. T., \& van der Hulst, J. M. 2007, ApJ, 655, L5

Bailin, J., \& Steinmetz, M. 2005, ApJ, 627, 647

Battaner, E., \& Jimenez-Vicente, J. 1998, A\&A, 332, 809

Battaner, E., Florido, E., \& Sanchez-Saavedra, M. L. 1990, A\&A, 236, 1

Battaner, E., Garrido, J. L., Sánchez-Saavedra, M. L., \& Florido, E. 1991, A\&A, 251, 402

Betancort-Rijo, J., \& Trujillo, I. 2008, in preparation

Bertin, G., \& Mark, J. W.-K. 1980, A\&A, 88, 289

Binney, J., 1981, MNRAS, 196, 455

Binney, J., Jiang, I.-G., \& Dutta, S. 1998, MNRAS, 297, 1237

Brunino, R., Trujillo, I., Pearce, F. R., \& Thomas, P. A. 2007, MNRAS, 375, 184 Dalcanton, J. J., \& Bernstein, R. A. 2002, AJ, 124, 1328

Dekel, A., \& Shlosman, I. 1983, in Intern. Kinemat. \& Dynam. Galaxies, IAU Symp., 100, 187

Florido, E., \& Battaner, E. 1997, A\&A, 327, 1

Fraternali, F., Binney, J., Oosterloo, T., \& Sancisi, R. 2007, New Astron. Rev., 51,95

García-Ruiz, I., Kuijken, K., \& Dubinski, J. 2002, MNRAS, 337, 459

Guijarro, A., Peletier, R., Battaner, E., et al. 2008, in preparation

Hahn, O., Porciani, C., Carollo, C. M., \& Dekel, A. 2007, MNRAS, 375, 489

Hunter, C., \& Toomre, A. 1969, ApJ, 155, 747

Jiang, I.-G., \& Binney, J. 1999, MNRAS, 303, 7

Kashikawa, N., \& Okamura, S. 1992, PASJ, 44, 493

Kuijken, K. 1991, ApJ, 376, 467

López-Corredoira, M., Betancort-Rijo, J. E., \& Beckman, J. E. 2002, A\&A, 386, 169

Mayor, M., \& Vigroux, L. 1981, A\&A, 98, 1

Navarro, J. F., Abadi, M. G., \& Steinmetz, M. 2004, ApJ, 613, L41

Nelson, R. W., \& Tremaine, S. 1995, MNRAS, 275, 897

Ostriker, E. C., \& Binney, J. J. 1989, MNRAS, 237, 785

Patiri, S., Cuesta, A. J., Prada, F., Betancort-Rijo, J., \& Klypin, A. 2006, ApJ, 652, L75

Paz, D., Stasyszyn, F., \& Padilla, N. 2008, [arXiv: 0804 . 4477]

Porciani, C., Dekel, A., \& Hoffman, Y. 2002, MNRAS, 332, 339

Quinn, T., \& Binney, J. 1992, MNRAS, 255, 729

Reshetnikov V., \& Combes F. 1998, A\&A, 337, 9

Reshetnikov, V., Battaner, E., Combes, F., \& Jiménez-Vicente, J. 2002, A\&A, 382,513

Revaz Y., \& Pfenninger D., 2001, in Gas and Galaxy Evolution, ed. J. E. Hibbard, M. Rupen, J. H. van Gorkom (San Francisco: Astronomical Society of the Pacific), ASP Conf. Proc., 240, 278

Saha, K., \& Jog, C. J., 2006, A\&A, 446, 897

Sánchez-Saavedra, M. L., Battaner, E, \& Florido, E. 1990, MNRAS, 246, 458

Sánchez-Saavedra, M. L., Battaner, E., Guijarro, A., López-Corredoira, M., \& Castro-Rodríguez, N. 2003, A\&A, 399, 457

Sánchez-Salcedo, F. 2006, MNRAS, 365, 555

Sparke, L. S., \& Casertano, S. 1988, MNRAS, 234, 873

Spergel, D. N., Bean, R., Dore, O., et al. 2007, ApJS, 170, 377

Toomre, A. 1983, in Internal kinematics and dynamics of galaxies (Dordrecht: D. Reidel Publishing Co.), 177

Trujillo, I., Carretero, C., \& Patiri, S. 2006, ApJ, 640, L111

van der Kruit, P. C. 2007, A\&A, 466, 883

Weinberg, M. D. 1998, MNRAS, 299, 499

Zurita, A., \& Battaner, E. 1997, A\&A, 322, 86 